\title{
Efeitos do agulhamento a seco no tratamento dos pontos-gatilho na síndrome dolorosa miofascial na coluna cervical: uma revisão sistemática de ensaios clínicos
}

\section{randomizados}

\author{
Effects of dry needling in the treatment of trigger points in cervical spine myofascial pain \\ syndrome: a systematic review of randomized clinical trials \\ Efectos de la punción seca en el tratamiento de los puntos gatillo en el síndrome de dolor miofascial \\ de la columna cervical: una revisión sistemática de ensayos clínicos aleatorizados
}

Recebido: 17/10/2021 | Revisado: 21/10/2021 | Aceito: 22/10/2021 | Publicado: 24/10/2021

\author{
Jéssica dos Santos Silva \\ ORCID: https://orcid.org/0000-0001-8791-0637 \\ Centro Universitário da Amazônia, Brasil \\ E-mail: jesssilva865@gmail.com \\ Gisele da Silva Cunha \\ ORCID: https://orcid.org/0000-0002-3582-0725 \\ Centro Universitário da Amazônia, Brasil \\ E-mail: giselecunha118@gmail.com \\ Bruna Caroline dos Santos Marques \\ ORCID: https://orcid.org/0000-0002-0393-8446 \\ Centro Universitário da Amazônia, Brasil \\ E-mail: brunamarques64@hotmail.com \\ Wanderson Fernandes Silva \\ ORCID: https://orcid.org/0000-0001-9389-5795 \\ Universidade do Estado do Pará, Brasil \\ E-mail: wandersonfsm@outlook.com
}

\begin{abstract}
Resumo
A síndrome dolorosa miofascial é uma das causas mais comuns de dor musculoesquelética e incapacidade acometendo uma faixa etária entre 31 e 50 anos, sendo mais propícia em pessoas que realizam movimentos repetitivos e com mais frequência na região cervical e cintura escapular. A etiologia exata da síndrome dolorosa miofascial não é totalmente definida porem seu tratamento é focado em diminuir a dor, melhorar a força muscular e proporcionar uma boa postura. $\mathrm{O}$ agulhamento a seco e um procedimento pouco invasivo, tendo por objetivo reduzir o quadro de dor miofascial ao colocar uma agulha diretamente sobre o ponto-gatilho e com a finalidade de provocar diminuição da dor local, além de restaurar a amplitude de movimento. Foi realizada uma revisão sistemática de ensaios clínicos randomizados, que tem por objetivo identificar os benefícios do agulhamento a seco na coluna cervical para pacientes com síndrome dolorosa miofascial através de uma revisão sistemática da literatura. Foram utilizadas as seguintes bases de dados: PEDro, ScieLo e Pubmed, nos idiomas português e inglês entre 2016 a 2021. Foram obtidos 552 estudos, após o cruzamento dos descritores e aplicação dos critérios de inclusão e exclusão, foram selecionados 10 artigos. O agulhamento a seco é um tratamento de grande valia para pacientes com síndrome de dor miofascial na coluna cervical, melhorando a dor. Porém, a combinação de outras técnicas produz relaxamento muscular, amplitude de movimento dos músculos cervicais refletindo na qualidade de vida destes pacientes.
\end{abstract}

Palavras-chave: Agulhamento seco; Pontos-gatilho; Síndromes da dor miofascial.

\begin{abstract}
Myofascial pain syndrome is one of the most common causes of musculoskeletal pain and disability, affecting an age group between 31 and 50 years, being more favorable in people who perform repetitive movements and more frequently in the cervical region and shoulder girdle. The exact etiology of myofascial pain syndrome is not fully defined, but its treatment is focused on decreasing pain, improving muscle strength and providing good posture. Dry needling is a minimally invasive procedure, aiming to reduce myofascial pain by placing a needle directly over the trigger point, aiming to reduce local pain, in addition to restoring range of motion. A systematic review of randomized clinical trials was carried out, aiming to identify the benefits of dry needling in the cervical spine for patients with myofascial pain syndrome through a literature review. The following databases were used: PEDro, ScieLo and Pubmed, in Portuguese and English between 2016 and 2021. A total of 552 studies were obtained, after crossing the descriptors and applying the inclusion and exclusion criteria, 10 articles were selected. Dry needling is a valuable
\end{abstract}


treatment for patients with myofascial pain syndrome in the cervical spine, improving pain. However, the combination of other techniques produces muscle relaxation, range of motion of the cervical muscles, reflecting on the quality of life of these patients.

Keywords: Dry needling; Trigger points; Myofascial pain syndromes.

\section{Resumen}

El síndrome de dolor miofascial es una de las causas más comunes de dolor y discapacidad musculoesquelética, afectando a un grupo de edad entre 31 y 50 años, siendo más favorable en personas que realizan movimientos repetitivos y con mayor frecuencia en la región cervical y cintura escapular. La etiología exacta del síndrome de dolor miofascial no está completamente definida, pero su tratamiento se centra en disminuir el dolor, mejorar la fuerza muscular y proporcionar una buena postura. La punción seca es un procedimiento mínimamente invasivo, cuyo objetivo es reducir el dolor miofascial colocando una aguja directamente sobre el punto gatillo, con el objetivo de reducir el dolor local, además de restaurar el rango de movimiento. Se realizó una revisión sistemática de ensayos clínicos aleatorizados, con el objetivo de identificar los beneficios de la punción seca en la columna cervical para pacientes con síndrome de dolor miofascial a través de una revisión de la literatura. Se utilizaron las siguientes bases de datos: PEDro, ScieLo y Pubmed, en portugués e inglés entre 2016 y 2021. Se obtuvieron un total de 552 estudios, tras cruzar los descriptores y aplicar los criterios de inclusión y exclusión, se seleccionaron 10 artículos. La punción seca es un tratamiento valioso para los pacientes con síndrome de dolor miofascial en la columna cervical, que mejora el dolor. Sin embargo, la combinación de otras técnicas produce relajación muscular, amplitud de movimiento de los músculos cervicales, reflejándose en la calidad de vida de estos pacientes.

Palabras clave: Punción seca; Puntos disparadores; Síndromes del dolor miofascial.

\section{Introdução}

A coluna cervical apresenta um alto grau de mobilidade e com pouca estabilidade, sendo assim ela torna-se mais suscetível a lesões. Dessa forma, a disfunção nessa região é uma condição comum afetando boa parte da população, que pode ser representada por um conjunto de sinais e sintomas envolvendo dor, limitação da amplitude dos movimentos fisiológicos, alteração da sensibilidade e dor à palpação dos músculos cervicais (Da Silva et al., 2020). Além disso, dor local ou referida, mobilidade restrita, fadiga e fraqueza muscular (Cabaleiro \& Magalhães 2020).

De acordo com Martín-Rodríguez et al., 2019, aproximadamente 70\% da população terá dor cervical pelo menos uma vez na vida levando a redução de atividades musculares da região e podendo causar alteração no controle motor cervical (CMC) em comparação com indivíduos saudáveis. A dor musculoesquelética de pescoço tem aumentado muito nos últimos anos, afetando $45 \%$ a $54 \%$ da população geral e sua prevalência é de $15 \%$ em homens e $23 \%$ em mulheres (Cabaleiro \& Magalhães 2020). Diante disso Arias-Buría et al., 2020, explica que a dor no pescoço tem uma vida e uma prevalência pontual quase tão alta como dor lombar e resulta em incapacidade substancial e custos para a sociedade. Cabaleiro \& Magalhães (2020) afirmar que a síndrome da dor miofascial é uma condição patológica extraordinariamente prevalente na população e que cerca de um terço dos pacientes com dor musculoesquelética atendem aos critérios de diagnóstico para síndrome dolorosa miofascial.

A síndrome dolorosa miofascial (SDM) é uma das causas mais comuns de dor musculoesquelética e incapacidade acometendo uma faixa etária entre 31 e 50 anos com maior número ao sexo feminino de meia-idade e sexo masculino na idade mais avançada, sendo mais propícia em pessoas que realizam movimentos repetitivos e com mais frequência na região cervical e cintura escapular (Fotana et al., 2018).

Segundo Ay et al., 2017, a etiologia exata da síndrome dolorosa miofascial não é totalmente definida porem seu tratamento é focado em diminuir a dor, melhorar a força muscular e proporcionar uma boa postura. Pelo contrario para Fotana et al., 2018, a dor cervical pode ser desencadeada por fatores sendo eles: Contusões, estiramentos, microtraumas, sobrecarga crônica ou uso excessivo dos músculos, desvia de coluna, má postura e más condições ergonômicas.

A SDM é uma condição caracterizada por dor crônica e associada a pontos-gatilho (PG) podendo ocorrer em um ou mais músculos (Ay et al., 2017). Segundo Arias-Buría et al., 2020, há evidencias que assuntos relacionado a dor no pescoço 
exibem vários distúrbios musculares, como exemplo a hiperatividade dos flexores superficiais do pescoço, os músculos esternocleidomastóideos e escalenos, essa hiperatividade pode levar a ativação dos pontos-gatilho nos músculos afetados.

Sanal Toprak e Ozsoy Unubo (2021), define o ponto-gatilho um nódulo neuromuscular em uma faixa tensa do musculo gerando uma disfunção pela sobrecarga incomum dos músculos. Logo, podem ser clinicamente classificados como ativos aqueles que induzem dor referida e que reproduz os sintomas, ou latentes, aqueles não responsáveis pelos sintomas sensoriais vivenciados (Arias-Buría et al., 2020). E sua compressão é dolorosa (Sanal Toprak \& Ozsoy Unubo, 2021). Os pontos gatilhos miofascial podem ter um efeito prejudicial nas pessoas, nas atividades sociais e no trabalho, e têm um impacto na qualidade de vida, dor e funcionalidade na área do pescoço e ombros (Ziaeifar et al., 2018).

As diretrizes de tratamento para dor cervical recomendam uma abordagem que consiste em mobilizações (torácica e cervical) em combinação com terapia de exercícios sendo eles: força, resistência, coordenação, propriocepção e treinamento de postural (Stieven et al., 2020). Segundo Fotana et al., 2018, dentre as técnicas utilizadas para o tratamento do ponto-gatilho, o agulhamento a seco (AS) é uma forma de tratamento não farmacológico que tem risco potencialmente eficaz para o tratamento da dor cervical.

Segundo Carvalho et al. (2017), agulhamento a seco, utilizado para tratamento da dor miofascial (DM), apesar de ser confundido com a acupuntura tradicional chinesa, é uma técnica ocidental baseada em princípios neurofisiológicos distintos, sendo descrito pela primeira vez por Travell na década de 1960.

O agulhamento a seco é uma técnica intervencionista, minimamente invasiva, utilizada no tratamento da dor miofascial (Carvalho et al., 2017). O agulhamento a seco é uma intervenção usada por fisioterapeutas para o gerenciamento de dor no pescoço (Gallego-Sendarrubias et al., 2021). Igualmente Fotana et al., 2018, define a técnica como um procedimento pouco invasivo, tendo por objetivo reduzir o quadro de dor miofascial ao colocar uma agulha diretamente sobre o ponto-gatilho e com a finalidade de provocar diminuição da dor local, além de restaurar a amplitude de movimento

As contraindicações absolutas do AS são: pessoas que tem fobia à agulha, áreas com linfedema, histórico de reação anormal a procedimentos anestésicos e estados de inconsciência, ou confusão mental. Já as contraindicações relativas são: terapia com anticoagulante, distúrbios vasculares, epilepsia, alergia ao metal da agulha, gravidez e em crianças (Carvalho et al., 2017). Dessa forma, o objetivo deste estudo é identificar os benefícios do agulhamento a seco na coluna cervical para pacientes com síndrome dolorosa miofascial através de uma revisão sistemática da literatura.

\section{Metodologia}

O presente estudo consiste em uma pesquisa de revisão sistemática que focaliza em uma questão definida, visando identificar, selecionar e avaliar as evidencia disponível (Galvão \& Pereira, 2014), de ensaios clínicos randomizados que consiste em um estudo experimental, realizados em seres humanos (De Souza, 2009), descritiva, pois expõem as características de uma determinada população, demandando técnicas padronizadas de coletas de dados (Prodanov \& de Freitas, 2013) com uma abordagem qualitativa mediante a descrição verbais (GIL, 2002), com objetivo de reunir uma síntese dos resultados de estudos sobre o tema proposto neste estudo.

Foram utilizadas as seguintes bases de dados: Physiotherapy Evidence Database (PEDro), Scientific Electronic Library Online (ScieLo), e National Library of Medicine (Pubmed), nos idiomas português e inglês, sendo selecionados artigos publicados no período de 2016 a 2021. Para estratégia de busca utilizamos os descritores em Ciências da Saúde (DECS): Agulhamento seco, Pontos-Gatilho, Sindromes da dor miofascial e seus equivalentes em inglês e espanhol.

Os artigos foram selecionados segundo os seguintes critérios de inclusão: estudos realizados em seres humanos do tipo ensaio clínicos randomizados, aplicação do agulhamento a seco, Pontos-Gatilho na Síndrome dolorosa miofascial, escore $\geq 5$ na escala PEDro. Os critérios para exclusão dos artigos foram: apresentar dor miofascial em outras regiões do corpo que 
não fossem em região cervical, ou não incluísse a técnica de agulhamento como um grupo de intervenção, trabalhos não concluídos e artigos que não é compatível com o tema.

Para evitar a inclusão excessiva de artigos, foram delimitadas as buscas nos seguintes campos: artigos que tivessem as palavras-chave pesquisadas no título e resumo publicados nos últimos 6 anos. A metodologia dos estudos selecionados foi avaliada pela escala PEDro, que é muito utilizada na área de reabilitação. Essa escala tem uma pontuação total de 10 pontos que avaliam a qualidade metodológica de estudos experimentais, sendo que escores $\geq 5$ são considerados de alta qualidade

\section{Resultados}

Na busca realizada em Outubro de 2021 nas plataformas de dados Pubmed, SciELO e PEDro, foram encontrados um total de 552 artigos. Após a leitura dos títulos, resumos e textos completos foram excluídos 542 por abordarem temas irrelevantes para a pesquisa, ou não preencherem os critérios de inclusão. Ao final um total de 10 artigos preencheram os critérios estabelecidos para esta revisão sistemática (Quadro 1). Dos artigos selecionados, todos apresentaram escores $\geq 5$, na escala de PEDro sendo considerados de alta qualidade. No Quadro 1 apresenta um breve resumo de 10 artigos utilizados, que evidenciam os efeitos do agulhamento a seco na síndrome dolorosa miofascial cervical. A população destes estudos consistiuse de 657 participantes com faixa etária entre 18 a 65 anos de idade. Nos 10 estudos, diversas intervenções terapêuticas foram abordadas comparando ao agulhamento a seco. As intervenções realizadas variaram de um dia de aplicação há seis semanas, porém, alguns artigos não especificam quantas sessões foram realizadas. Não foi relatado nos estudos o tempo de aplicação de agulhamento a seco e de outras técnicas realizadas em cada sessão.

Quadro 1 - Resultados dos estudos entre os anos de 2016 à 2021.

\begin{tabular}{|c|c|c|c|c|c|c|}
\hline Título & Autor/Ano & Amostra & Idade & Objetivo & Resultados & $\begin{array}{c}\text { Escore } \\
\text { PEDRO }\end{array}$ \\
\hline $\begin{array}{l}\text { Comparing trigger point } \\
\text { dry needling and manual } \\
\text { pressure technique for the } \\
\text { management of } \\
\text { myofascial neck/shoulder } \\
\text { pain: A randomized } \\
\text { clinical trial. }\end{array}$ & $\begin{array}{c}\text { DE } \\
\text { MEULEMEE } \\
\text { STER, K. E. } \\
\text { et al., } 2017 .\end{array}$ & 42 & $\begin{array}{c}24 \text { a } 54 \\
\text { anos }\end{array}$ & $\begin{array}{c}\text { Determinar se o } \\
\text { agulhamento a seco tem } \\
\text { melhores efeitos sobre a } \\
\text { deficiência, dor e } \\
\text { características musculares } \\
\text { em tratamento da dor } \\
\text { miofascial no } \\
\text { pescoço/ombro em } \\
\text { mulheres. }\end{array}$ & $\begin{array}{l}\text { Ambas as técnicas de } \\
\text { tratamento levam a efeitos } \\
\text { de tratamento de curto e } \\
\text { longo prazo. O agulhamento } \\
\text { seco não foi mais eficaz do } \\
\text { que a pressão manual no } \\
\text { tratamento da dor } \\
\text { miofascial no pescoço / } \\
\text { ombro. }\end{array}$ & 7 \\
\hline $\begin{array}{l}\text { The effectiveness of dry } \\
\text { needling and exercise } \\
\text { therapy in patients with } \\
\text { dizziness caused by } \\
\text { cervical myofascial pain } \\
\text { syndrome; prospective } \\
\text { randomized clinical } \\
\text { study. }\end{array}$ & $\begin{array}{l}\text { AYDIN, T. et } \\
\text { al., } 2018\end{array}$ & 61 & $\begin{array}{c}\text { Mais ou } \\
\text { menos } \\
38 \text { anos }\end{array}$ & $\begin{array}{l}\text { Comparar a eficácia da } \\
\text { terapia com agulhas secas } \\
\text { combinada com exercícios e } \\
\text { apenas o tratamento com } \\
\text { exercícios para aliviar a } \\
\text { tontura causada pela } \\
\text { síndrome da dor miofascial } \\
\text { cervical. }\end{array}$ & $\begin{array}{l}\text { Tanto a terapia com agulhas } \\
\text { secas + exercícios quanto à } \\
\text { terapia com exercícios } \\
\text { isoladamente foram eficazes } \\
\text { no tratamento da tontura } \\
\text { causada pela síndrome da } \\
\text { dor miofascial cervical. }\end{array}$ & 5 \\
\hline $\begin{array}{l}\text { Kinesio taping versus dry } \\
\text { needling in the treatment } \\
\text { of myofascial pain of the } \\
\text { upper trapezius muscle: } \\
\text { A randomized, single } \\
\text { blind (evaluator), } \\
\text { prospective study. }\end{array}$ & $\begin{array}{l}\text { DOGAN, N. } \\
\text { et al., } 2019\end{array}$ & 42 & $\begin{array}{c}20 \text { a } 50 \\
\text { anos }\end{array}$ & $\begin{array}{l}\text { Comparar a eficácia dos } \\
\text { métodos Kinesio Taping e } \\
\text { agulhamento seco em } \\
\text { pacientes com síndrome de } \\
\text { dor miofascial relacionada } \\
\text { ao ponto-gatilho do } \\
\text { músculo trapézio superior. }\end{array}$ & $\begin{array}{l}\text { Kinesio Taping pode ser } \\
\text { uma opção de inativação do } \\
\text { ponto de gatilho em } \\
\text { pacientes que não desejam } \\
\text { ser agulhados ou que } \\
\text { apresentam contraindicação } \\
\text { para outros tratamentos } \\
\text { além do Kinesio Taping. }\end{array}$ & 5 \\
\hline $\begin{array}{l}\text { The effects of shock } \\
\text { wave and dry needling on } \\
\text { active trigger points of } \\
\text { upper trapezius muscle in } \\
\text { patients with non-specific }\end{array}$ & $\begin{array}{c}\text { MANAFNEZ } \\
\text { HAD, J. et al., } \\
2019\end{array}$ & 70 & 38 & $\begin{array}{l}\text { Comparar os efeitos da } \\
\text { terapia por ondas de choque } \\
\text { extracorpóreas com técnicas } \\
\text { de agulhamento seco sobre } \\
\text { os pontos de gatilho do }\end{array}$ & $\begin{array}{l}\text { Agulhamento a seca e } \\
\text { terapia por ondas de choque } \\
\text { extracorpóreas têm efeitos } \\
\text { semelhantes na redução dos } \\
\text { sintomas da dor de ponto }\end{array}$ & 6 \\
\hline
\end{tabular}




\begin{tabular}{|c|c|c|c|c|c|c|}
\hline $\begin{array}{l}\text { neck pain: A randomized } \\
\text { clinical trial. }\end{array}$ & & & & $\begin{array}{l}\text { músculo trapézio superior } \\
\text { em pacientes com dor } \\
\text { cervical inespecífica. }\end{array}$ & $\begin{array}{c}\text { gatilho do músculo trapézio } \\
\text { e capacidade funcional em } \\
\text { pacientes com dor cervical } \\
\text { inespecífica. }\end{array}$ & \\
\hline $\begin{array}{l}\text { Effects of dry needling in } \\
\text { the sternocleidomastoid } \\
\text { muscle on cervical motor } \\
\text { control in patients with } \\
\text { neck pain: A randomised } \\
\text { clinical trial. }\end{array}$ & $\begin{array}{l}\text { MARTÍN- } \\
\text { RODRÍGUEZ } \\
\text {, A. et al., } \\
2019\end{array}$ & 34 & $\begin{array}{l}>18 \\
\text { anos }\end{array}$ & $\begin{array}{l}\text { Determinar as alterações } \\
\text { produzidas pelo } \\
\text { agulhamento seco do ponto } \\
\text { de gatilho do musculo } \\
\text { esternocleidomastóideo em } \\
\text { pacientes com dor cervical e } \\
\text { observar como isso pode } \\
\text { modificar o controle motor } \\
\text { cervical. }\end{array}$ & $\begin{array}{l}\text { Os efeitos do agulhamento } \\
\text { seco dentro e fora dos } \\
\text { pontos-gatilho miofasciais } \\
\text { ativos não diferiram neste } \\
\text { estudo. Especificamente } \\
\text { uma redução da dor no } \\
\text { pescoço e um aumento do } \\
\text { Controle Motor Cervical. }\end{array}$ & 8 \\
\hline $\begin{array}{l}\text { Efficacy of dry needling } \\
\text { as an adjunct to manual } \\
\text { therapy for patients with } \\
\text { chronic mechanical neck } \\
\text { pain: A randomised } \\
\text { clinical trial. }\end{array}$ & $\begin{array}{l}\text { GALLEGO- } \\
\text { SENDARRU } \\
\text { BIAS, G. M. } \\
\text { et al., } 2020\end{array}$ & 101 & $\begin{array}{l}\text { Entre } 18 \\
\text { a } 55 \\
\text { anos }\end{array}$ & $\begin{array}{l}\text { Comparamos o efeito do } \\
\text { agulhamento seco } \\
\text { combinado com terapia } \\
\text { manual na dor, limiar de } \\
\text { pressão de dor, amplitude } \\
\text { de movimento cervical e } \\
\text { deficiência do pescoço em } \\
\text { pacientes com mecânica } \\
\text { crônica dor de pescoço. }\end{array}$ & $\begin{array}{c}\text { Agulhamento seco + terapia } \\
\text { manual é eficaz e } \\
\text { significativamente melhor } \\
\text { do que agulhamento seco } \\
\text { simulado + terapia manual } \\
\text { na redução da intensidade } \\
\text { da dor, limiar de dor à } \\
\text { pressão, deficiência cervical } \\
\text { e amplitude de movimento } \\
\text { cervical em pacientes com } \\
\text { dor cervical mecânica } \\
\text { crônica. }\end{array}$ & 7 \\
\hline $\begin{array}{l}\text { Short-Term effects of } \\
\text { PENS versus dry } \\
\text { needling in subjects with } \\
\text { unilateral mechanical } \\
\text { neck pain and active } \\
\text { myofascial trigger points } \\
\text { in levator scapulae } \\
\text { muscle: A randomized } \\
\text { controlled trial }\end{array}$ & $\begin{array}{l}\text { GARCIA-DE- } \\
\text { MIGUEL, S. } \\
\text { et al., } 2020\end{array}$ & 44 & $\begin{array}{c}=/>18 \\
\text { anos }\end{array}$ & $\begin{array}{l}\text { Investigar se a estimulação } \\
\text { elétrica nervosa percutânea } \\
\text { é mais eficaz do que o } \\
\text { agulhamento seco em curto } \\
\text { prazo em indivíduos com } \\
\text { dor cervical mecânica. }\end{array}$ & $\begin{array}{c}\text { A estimulação elétrica } \\
\text { nervosa percutânea parece } \\
\text { produzir maiores melhorias } \\
\text { na sensibilidade e } \\
\text { incapacidade do que a } \\
\text { agulhamento seco no } \\
\text { seguimento de curto prazo. }\end{array}$ & 7 \\
\hline $\begin{array}{l}\text { Dry needling combined } \\
\text { with guideline-based } \\
\text { physical therapy provides } \\
\text { no added benefit in the } \\
\text { management of chronic } \\
\text { neck pain: A randomized } \\
\text { controlled trial. }\end{array}$ & $\begin{array}{l}\text { STIEVEN, F. } \\
\text { F. et al., } 2020\end{array}$ & 116 & $\begin{array}{c}18 \text { a } 65 \\
\text { anos }\end{array}$ & $\begin{array}{l}\text { Determinar o benefício } \\
\text { adicional de combinar } \\
\text { agulhas secas com um } \\
\text { programa de tratamento de } \\
\text { fisioterapia baseado em } \\
\text { diretrizes que consiste em } \\
\text { exercícios e terapia manual } \\
\text { para dor e deficiência em } \\
\text { pessoas com dor cervical } \\
\text { crônica. }\end{array}$ & $\begin{array}{c}\text { Quando combinado com } \\
\text { fisioterapia baseada em } \\
\text { diretrizes para dor no } \\
\text { pescoço, agulhas secas } \\
\text { resultaram em pequenas } \\
\text { melhorias na dor. }\end{array}$ & 8 \\
\hline $\begin{array}{l}\text { Effects of pain } \\
\text { neuroscience education } \\
\text { and dry needling for the } \\
\text { management of patients } \\
\text { with chronic myofascial } \\
\text { neck pain: A randomized } \\
\text { clinical trial. }\end{array}$ & $\begin{array}{l}\text { VALIENTE- } \\
\text { CASTRILLO, } \\
\text { P. et al., } 2020\end{array}$ & 60 & $\begin{array}{c}18 \text { a } 65 \\
\text { anos }\end{array}$ & $\begin{array}{l}\text { Observar os efeitos da dor, } \\
\text { incapacidade e fatores } \\
\text { psicológicos de ponto de } \\
\text { gatilho miofascial, } \\
\text { agulhamento seco, com } \\
\text { educação em neurociência } \\
\text { da dor, agulhamento seco } \\
\text { sozinho, cuidado de } \\
\text { controle como usual em } \\
\text { pacientes com dor cervical } \\
\text { crônica. }\end{array}$ & $\begin{array}{l}\text { Agulhamento seco sozinho } \\
\text { foi mais eficaz na redução } \\
\text { da dor cervical inespecífica } \\
\text { crônica e incapacidade do } \\
\text { que cuidado de controle } \\
\text { como usual no } \\
\text { acompanhamento. }\end{array}$ & 8 \\
\hline $\begin{array}{l}\text { Comparison of dry } \\
\text { needling and kinesio } \\
\text { taping methods in the } \\
\text { treatment of myofascial } \\
\text { pain syndrome: A single } \\
\text { blinded randomised } \\
\text { controlled study. }\end{array}$ & $\begin{array}{l}\text { YASAR, M. } \\
\text { F. et al., } 2021\end{array}$ & 87 & $\begin{array}{c}20 \text { e } 60 \\
\text { anos }\end{array}$ & $\begin{array}{l}\text { omparar a eficácia do } \\
\text { kinesio taping e do } \\
\text { agulhamento seco no } \\
\text { tratamento da síndrome da } \\
\text { dor miofascial do músculo } \\
\text { trapézio. }\end{array}$ & $\begin{array}{l}\text { No tratamento da síndrome } \\
\text { da dor miofascial, adicionar } \\
\text { agulhamento seco ou } \\
\text { Kinesio taping ao programa } \\
\text { de exercícios pode fornecer } \\
\text { contribuições importantes } \\
\text { para o tratamento. }\end{array}$ & 7 \\
\hline
\end{tabular}




\section{Discussão}

Dentre os vários recursos disponíveis para o tratamento da dor foi realizado um estudo de revisão sistemática de ensaios clínicos randomizados voltados para técnica de agulhamento a seco, enfatizando os benefícios dessa técnica no tratamento da síndrome dolorosa miofascial da coluna cervical.

De Meulemeester et al. (2017) realizou um estudo randomizado em funcionárias de um escritório comparando agulhamento a seco e pressão manual na dor no pescoço e ombro de origem miofascial. Foram avaliadas 42 funcionárias, dos quais 22 foram alocados para o grupo técnica de pressão manual e 20 sujeitos foram incluídos no grupo de agulhamento a seco, durante a execução do estudo 4 pacientes desistiram. 14\% dos pontos gatilho foram encontrados no trapézio superior esquerdo e $16 \%$ no direito, $11 \%$ no musculo elevador da escapula (direito) e $11 \%$ no musculo trapézio médio (direito). Após o estudo foi observado que o agulhamento a seco não foi mais eficaz do que a pressão manual no tratamento de dor no pescoço e ombro de origem miofascial nas funcionárias do escritório, porém após as 4 semanas do programa de tratamento, observou-se a melhora no limiar de dor de pressão mais alto, elasticidade muscular e rigidez. Diferentemente do estudo de Gallego-Sendarrubias et al., 2020 onde participaram um total de 101 pacientes, do qual 47 foram atribuídos ao grupo de agulhamento a seco associado com a terapia manual e 54 para o grupo de agulhamento a seco. Sobre os efeitos os dois grupos geraram resultados significativos em termos de intensidade da dor, limiar de dor a pressão e incapacidade, após duas intervenções e com seguimento de curto prazo.

Em outro estudo, Aydin et al., 2018, realizou um estudo com 61 pacientes, sendo dividido em dois grupos. No primeiro grupo, 30 pacientes foram randomizados sem exercício e no segundo grupo, 31 pacientes foram submetidos ao agulhamento a seco mais exercícios, um total de 55 pacientes completou o estudo. Na pesquisa observou-se que o agulhamento a seco associado com a terapia por exercício e somente a terapia por exercício, só foram eficazes no tratamento de tonturas causadas por síndrome da dor miofascial cervical. Já no estudo de Stieven et al., 2020, foram incluídos 116 participantes na pesquisa e após um mês depois da randomização 18 participantes que foram submetidos ao agulhamento a seco associado à fisioterapia obtiveram uma redução pequena na intensidade da dor 1 dia antes e 21 semanas antes, observou-se também que não houve nenhum efeito na colocação do agulhamento a seco associado à fisioterapia em 1 mês após a randomização e quando associado à fisioterapia voltada para a dor cervical crônica, o agulhamento a seco proporcionou pequenas melhorias, não sendo clinicamente significativas, na dor em curto prazo.

Além disso, na pesquisa de Doğan et al. (2019), foi possível observar que o agulhamento a seco seguido de alongamento foi considerado mais eficaz do que o alongamento sozinho, e que o kinesio taping pode ser uma escolha relevante para tratamento de pontos gatilho na dor miofascial em pacientes que discordam serem submetidos a agulhadas ou que tenham alguma contraindicação para outros tratamentos, incluindo agulhamento a seco. Observou-se também que o Kinesio Taping juntamente com o agulhamento a seco mostrou ter efeitos igualmente positivos no tratamento de pontos gatilho na dor miofascial do músculo trapézio superior, especialmente na redução da dor, nessa pesquisa foram incluídos 42 pacientes. Em contrapartida na pesquisa de Yasar et al., 2021, foram selecionados 88 pacientes para participar do estudo, do qual 26 pacientes foram distribuídos aleatoriamente no grupo de kinesio taping, 32 no grupo de agulhamento a seco e 30 no grupo controle. Observou-se bons resultados com associação de exercícios convencionais e aplicações com tratamento de kinesio taping e agulhamento a seco.

Segundo o estudo de Martín-Rodríguez et al., 2019, foram identificados dor cervical inespecífica em 69 pacientes, e após o estudo observou-se que o agulhamento a seco no ponto de gatilho dentro e fora do musculo esternocleidomastóideo gerar uma redução da dor no pescoço e aumento do controle motor cervical, no entanto, esses achados devem ser interpretados com cautela devido à falta de um grupo de controle. 
Todavia, Manafnezhad et al., 2019, submeteu ao estudo 70 participantes, sendo dividido 35 para o grupo de agulhamento a seco e 35 pacientes ao grupo de terapia por ondas de choque extracorpórea. Constatou-se no final do estudo que os efeitos do agulhamento a seco e terapia por ondas de choque extracorpóreas são semelhantes na redução dos sintomas da dor no ponto gatilho do músculo trapézio e na capacidade funcional em pacientes com dor cervical inespecífica.

Ademais no estudo de Valiente-Castrillo et al., 2020, foram selecionados 120 pacientes com dor miofascial cervical, dos quais 60 pacientes concluíram com sucesso o protocolo do estudo. Especificamente, 19 indivíduos completaram o estudo no grupo controle de tratamento usual, 20 no Grupo agulhamento a seco em pontos-gatilho, e 21 no grupo agulhamento seco em pontos-gatilho e educação neurociência da dor, nos grupos de agulhamento seco em pontos gatilho, $17 \%$ receberam agulhamento unilateralmente e $83 \%$ bilateralmente. Durante o acompanhamento do estudo, observaram-se melhorias consideráveis na dor crônica no pescoço em todos os grupos.

Por fim, conforme a pesquisa de Garcia-de-Miguel et al., 2020, foram submetidos 44 participantes, sendo atribuídos 22 pacientes ao grupo agulhamento a seco e os outros 22 no grupo de estimulação elétrica nervosa percutânea. Nos resultados de acompanhamento de curto prazo, observou-se que a estimulação elétrica nervosa percutânea produziu maiores melhorias na sensibilidade e incapacidade do que o agulhamento a seco. Entretanto, foi possível perceber que não houve nenhuma diferença entre os grupos em relação à intensidade da dor e força de flexão lateral e teve inconsistências em relação à amplitude de movimento. Contudo pesquisas melhores sobre acompanhamento de médio e longo prazo são necessárias para avaliar as possíveis diferenças entre esses tratamentos ao longo do tempo.

\section{Considerações Finais}

De modo geral, pode-se observar que a síndrome dolorosa miofascial é considerada um problema de saúde, sendo uma das causas mais comuns de incapacidade, limitação e dor em pacientes que apresentam algias desta natureza. Com base nos estudos controlados e randomizados, foi evidenciado que a técnica de agulhamento a seco demonstra-se eficaz no tratamento da diminuição da dor de origem miofascial. Porem nota-se um ganho adicional na associação de outras técnicas como pressão manual, exercícios terapêuticos, alongamento, kinesio taping, terapia por ondas de choque extracorpórea, terapia manual e estimulação elétrica nervosa percutânea. As associações das técnicas apresentaram uma melhora significativa na intensidade da dor, ganho de amplitude de movimento dos músculos cervicais, por conseguinte, melhora na qualidade de vida dos indivíduos. Contudo, é importante a realização de mais estudos de ensaios clínicos com amostras mais controladas, e a associação do agulhamento a seco com outras técnicas terapêuticas para aumentar os conhecimentos científicos acerca da técnica.

\section{Agradecimentos}

Agradecemos a Deus, pela dadiva da vida, e por nos permitir ultrapassar todos os obstáculos encontrados ao longo da realização deste trabalho, nossas famílias pelo apoio durante toda a vida, e nosso orientador Wanderson Fernandes Silva pela dedicação e paciência durante todo o processo.

\section{Referências}

Arias-Buría, J. L., Monroy-Acevedo, Á., Fernández-de-las-Peñas, C., Gallego-Sendarrubias, G. M., Ortega-Santiago, R., \& Plaza-Manzano, G. (2020). Effects of dry needling of active trigger points in the scalene muscles in individuals with mechanical neck pain: A randomized clinical trial. Acupuncture in Medicine, 38(6), 380-387. https://doi.org/10.1177/0964528420912254

Ay, S., Konak, H. E., Evcik, D., \& Kibar, S. (2017). The effectiveness of Kinesio Taping on pain and disability in cervical myofascial pain syndrome. Revista Brasileira De Reumatologia (English Edition), 57(2), 93-99. https://doi.org/10.1016/j.rbre.2016.03.012

Aydın, T., Dernek, B., Sentürk Ege, T., Karan, A., \& Aksoy, C. (2018). The effectiveness of dry needling and exercise therapy in patients with dizziness caused by cervical myofascial pain syndrome; prospective randomized clinical study. Pain Medicine, 20(1), 153-160. https://doi.org/10.1093/pm/pny072 
Cabaleiro, P., J., R.., \& Magalhães, A. (2020). Compressão Isquémica vs. Agulhamento Seco no Tratamento da Cervicalgia Associada à Síndrome de Dor Miofascial: Revisão da Literatura. Universidade Fernando pessoa fcs/ess licenciatura em fisioterapia projeto de estagio profissionalizante II. http://hdl.handle.net/10284/9188

Carvalho, A. V. d., Grossmann, E., Ferreira, F. R., Januzzi, E., \& Fonseca, R. M. D. F. B. (2017). The use of dry needling in the treatment of cervical and masticatory myofascial pain. Revista Dor, 18(3). https://doi.org/10.5935/1806-0013.20170111

Da Silva, T., Oliveira, A. L., Dos Santos, S. M., Oliveira, T. d. J., Guimarães, L. S., Ferreira, A. M. R., Ramos, S. D. d. N., Silva, R. T. B., Cruz, T. T. d., Rodrigues dos Santos, K. P., Vieira, L. C., Lima, V. M. d., Coutinho, E. N., Carvalho, D. F. d., \& Ferreira, C. P. (2020). Qualidade de vida e prevalência de dor na região cervical em acadêmicos. Revista Eletrônica Acervo Saúde, (39), Artigo e2021. https://doi.org/10.25248/reas.e2021.2020

De Meulemeester, K. E., Castelein, B., Coppieters, I., Barbe, T., Cools, A., \& Cagnie, B. (2017). Comparing trigger point dry needling and manual pressure technique for the management of myofascial neck/shoulder pain: A randomized clinical trial. Journal of Manipulative and Physiological Therapeutics, 40(1), 11-20. https://doi.org/10.1016/j.jmpt.2016.10.008

De Souza, R., F. (2009). O que é um estudo clínico randomizado? Medicina (Ribeirão Preto);42(1): 3-8

Doğan, N., Şengül, İ., Akçay-Yalbuzdağ, Ş., \& Kaya, T. (2019). Kinesio taping versus dry needling in the treatment of myofascial pain of the upper trapezius muscle: A randomized, single blind (evaluator), prospective study. Journal of Back and Musculoskeletal Rehabilitation, 32(5), 819-827. https://doi.org/10.3233/bmr-181162

Fontana, F., D., da Silva Araujo, R., \& Franciscatto Stieven, F. (2018). O agulhamento a seco no tratamento da dor miofascial em pontos-gatilho na coluna cervical: uma revisão sistemática de ensaios clínicos randomizados. movimento \& saúde $\bullet$ Revista Inspirar, $17(3), 10$.

Gallego-Sendarrubias, G. M., Rodríguez-Sanz, D., Calvo-Lobo, C., \& Martín, J. L. (2020). Efficacy of dry needling as an adjunct to manual therapy for patients with chronic mechanical neck pain: A randomised clinical trial. Acupuncture in Medicine, 38(4), 244-254. https://doi.org/10.1136/acupmed-2018011682

Gallego-Sendarrubias, G. M., Voogt, L., Arias-Buría, J. L., Bialosky, J., \& Fernández-de-las-Peñas, C. (2021). Can patient expectations modulate the shortterm effects of dry needling on sensitivity outcomes in patients with mechanical neck pain? A randomized clinical trial. Pain Medicine. https://doi.org/10.1093/pm/pnab134

Galvão, T. F., \& Pereira, M. G. (2014). Revisões sistemáticas da literatura: Passos para sua elaboração. Epidemiologia e Serviços de Saúde, 23(1), 183-184. https://doi.org/10.5123/s1679-49742014000100018

Garcia-de-Miguel, S., Pecos-Martin, D., Larroca-Sanz, T., Sanz-de-Vicente, B., Garcia-Montes, L., Fernandez-Matias, R., \& Gallego-Izquierdo, T. (2020). Short-Term effects of PENS versus dry needling in subjects with unilateral mechanical neck pain and active myofascial trigger points in levator scapulae muscle: A randomized controlled trial. Journal of Clinical Medicine, 9(6), 1665. https://doi.org/10.3390/jcm9061665

Gil, A. C. (2002). Como elaborar projetos de pesquisa (4a ed.). ATLAS S.A.

Manafnezhad, J., Salahzadeh, Z., Salimi, M., Ghaderi, F., \& Ghojazadeh, M. (2019). The effects of shock wave and dry needling on active trigger points of upper trapezius muscle in patients with non-specific neck pain: A randomized clinical trial. Journal of Back and Musculoskeletal Rehabilitation, 32(5), 811818. https://doi.org/10.3233/bmr-181289

Martín-Rodríguez, A., Sáez-Olmo, E., Pecos-Martín, D., \& Calvo-Lobo, C. (2019). Effects of dry needling in the sternocleidomastoid muscle on cervical motor control in patients with neck pain: A randomised clinical trial. Acupuncture in Medicine, 37(3), 151-163. https://doi.org/10.1177/0964528419843913

Prodanov, C. C., \& de Freitas, E. C. (2013). Metodologia do trabalho cientifico: Métodos e técnicas da pesquisa e do trabalho acadêmico (2a ed.). Feevale.

Sanal Toprak, C., \& Ozsoy Unubo, T. (2021). Effectiveness of dry needling in the treatment of neck pain and disability associated with myofascial trigger points. Marmara Medical Journal. https://doi.org/10.5472/marumj.866557

Stieven, F. F., Ferreira, G. E., Wiebusch, M., de Araújo, F. X., da Rosa, L. H. T., \& Silva, M. F. (2020). Dry needling combined with guideline-based physical therapy provides no added benefit in the management of chronic neck pain: A randomized controlled trial. Journal of Orthopaedic \& Sports Physical Therapy, 50(8), 447-454. https://doi.org/10.2519/jospt.2020.9389

Stieven, F. F., Ferreira, G. E., Wiebusch, M., de Araújo, F. X., da Rosa, L. H. T., \& Silva, M. F. (2020). Dry needling combined with guideline-based physical therapy provides no added benefit in the management of chronic neck pain: A randomized controlled trial. Journal of Orthopaedic \& Sports Physical Therapy, 50(8), 447-454. https://doi.org/10.2519/jospt.2020.9389

Valiente-Castrillo, P., Martín-Pintado-Zugasti, A., Calvo-Lobo, C., Beltran-Alacreu, H., \& Fernández-Carnero, J. (2020). Effects of pain neuroscience education and dry needling for the management of patients with chronic myofascial neck pain: A randomized clinical trial. Acupuncture in Medicine, 096452842092030. https://doi.org/10.1177/0964528420920300

Yasar, M. F., Yaksi, E., Kurul, R., Alisik, T., \& Seker, Z. (2021). Comparison of dry needling and kinesio taping methods in the treatment of myofascial pain syndrome: A single blinded randomised controlled study. International Journal of Clinical Practice. https://doi.org/10.1111/ijcp.14561

Ziaeifar, M., Arab, A. M., Mosallanezhad, Z., \& Nourbakhsh, M. R. (2018). Dry needling versus trigger point compression of the upper trapezius: A randomized clinical trial with two-week and three-month follow-up. Journal of Manual \& Manipulative Therapy, 27(3), 152-161. https://doi.org/10.1080/10669817.2018.1530421 